\title{
Primary Lateral Sclerosis, Report of a Case and Bibliography Revision
}

\author{
Miranda Nava Gabriel ${ }^{1 *}$ and García García Luis Gilberto ${ }^{2}$ \\ ${ }^{1}$ Head of the Neurology Service of the Regional Military Hospital of Guadalajara, Mexico
}

${ }^{2}$ Physician of the regional military specialty Hospital of Guadalajara, Mexico

*Corresponding author: Miranda Nava Gabriel, Head of the Neurology Service of the Regional Military Hospital of Guadalajara, Mexico

\begin{abstract}
Introduction: Primary lateral sclerosis is a rare disease involving the upper motor neuron, producing a bulbospinal spasticity. The course of the disease is insidious and progressive, usually starting with the lower extremities, and later becoming a tetrapyremidal syndrome. As a rare disease, diagnosis in most cases is exclusionary, and the patient should be studied extensively, clinically, including a thorough medical history, laboratorial and with the relevant cabinet studies.

Classic Case: It is male patient who starts his clinical picture about a year ago with weakness in left pelvic limb, subsequently accompanied by pain and paresthesias, manifesting the same symptomatology later in the contralateral leg and upper left limb. Currently is added index and middle toe hypoesthesia, moderate tremor in left arm, with the contrast of the middle toe over the ring of said hand. It has an inability to lift light objects for short periods of time, as well as fatigue in short periods of time when performing daily activities, which greatly limits their daily life.

Conclusion: Motor neurone diseases are divided into two groups, and in the case studied, the upper motor neuron is exclusively affected. As it is a rare disease, with a low incidence, multiple differential diagnoses will be considered before concluding in it, considering it a diagnosis of exclusion. The natural history of the disease will always have a grim outcome, with poor prognosis for life and function, despite the measures taken to change the course of the disease.
\end{abstract}

Keywords: Motoneuron; upper; spastic; resonance

\section{Introduction}

Primary lateral sclerosis is a rare disease involving the upper motoneuron, which is characterized by a progressive bulbospinal spasticity, with selective degeneration of pyramidal neurons located in the precentral convolution [1]. Primary lateral sclerosis makes up approximately $1 \%$ to $4 \%$ of all patients with motor neuron diseases [2]. The onset of the disease is insidious, with a slow and progressive spastic paralysis, usually starting at the lower extremities, and then becoming a tetrapyremidal syndrome. On average, patients are estimated to have a life prognosis greater than 10 years from the onset of symptoms. Hyperreflexia, moderate weakness, dizziness, lack of coordination may also occur within the clinical picture. In the physical examination you can find spasticity, increased osteotendinous reflexes, and as a predominant sign, stiffness [2,3]. Diagnosis is usually performed clinically, relying on MAGNETIC resonance imaging, among other studies, to rule out other pathological entities, showing in this study a marked atrophy in the primary motor cortex [3]. In some studies, diffusion magnetic resonance imaging has been used, showing a functional increase in cerebral axonal activity, especially the brain-cerebellar, which could explain an adaptive process through functional neuroplasticity, however, the prognosis of the disease remains unfavorable and irreversible despite these changes [4]. Although there are some diagnostic criteria for the disease, as it is a rare entity, not all inclusions for it are always met, so it is usually a diagnosis of exclusion [5,6] (Figure 1). 


\section{Diagnostic criteria proposed by Pringle and Cols.}

- Clinical:

1.- Insidious onset of spastic paresis, usually starting in lower extremities, but occasionally in bulbar system or upper extremities.

2.- Start in adulthood, usually in the fifth decade of life or after.

3.- Absence of family history.

4.- Gradually progressive course.

5.- Duration greater than or equal to three years.

6.- Clinical findings generally limited to dysfunction of the corticospinal system.

7.- Symmetrical distribution, with final development of severe spinobulbar spastic paresis.

- Laboratory (for differential diagnosis).

1.- Blood chemistry in normal serum including vitamin B12 levels.

2.- Negative serology for syphilis and in endemic areas for Lyme disease and HTLV-1 virus.

3.- Normal CSF parameters including absence of oligoclonal bands.

4.- In most patients lack potential straining in GMS, occasional fibrillation and increased insertional activity in few muscles. Absence of compressive lesions of the cervical marrow or foramen magno in images of MRI-

- In addition to primary lateral sclerosis.

1.-Preserved bladder function.

2.- Absence or very prolonged latency in cortical evoked motor responses in the presence of normal peripheral evoked stimuli combined with potentials of muscle action.

3.-Focal atrophy of the pre-central rotation in MRI.

4.- Decreased glucose consumption in the pericentral region in PET.

Figure 1: Diagnostic criteria proposed by Pringle and Cols.

\section{Clinical Case}

They are a 42-year-old male patient, who started his clinical picture about a year ago, when when he is parading, I notice some weakness in left pelvic limb, progressing over the course of the days to diffuse pain and local paresthesias, and subsequently having the same problem in the contralateral leg and then to upper left limb throughout, progressively to date. Currently, adding to the above, it has hypoesthesia of index and middle toe, stiffness in all the arcs of the movement of the affected limbs, moderate tremor in the left arm, with the oversetting of the middle toe over the ring of said hand. Symptomatology is exacerbated in the mornings. It has an inability to lift light objects for short periods of time, as well as fatigue in short periods of time when performing daily activities, which greatly limits their daily life. It denies important here family backgrounds, as well as surgeries, drug addictions, or known diseases (Figure 2).

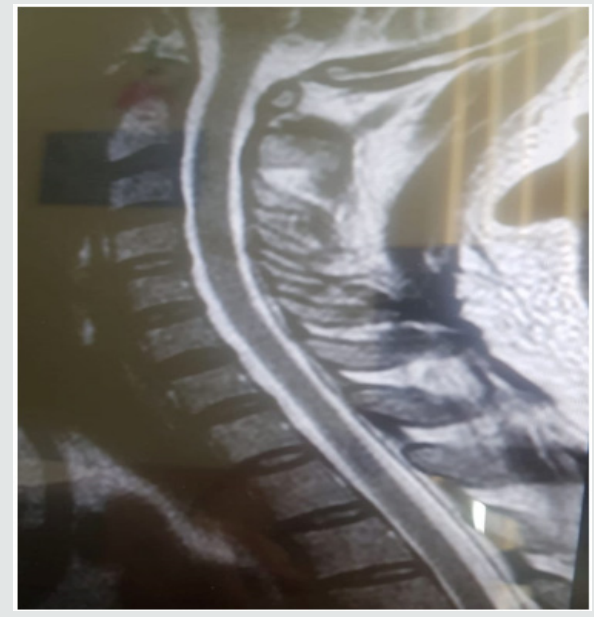

Figure 2: Cervical spinal MRI where you see a normal morphology without compromise spinal cord. 


\section{Discussion}

Primary lateral sclerosis is a rare disease affecting the upper motor motoneuron of the primary motor area (pre-central circumvolution),characterized by having an insidious onset in people with no known risk factors, with a clinical picture in which a spastic paresis of lower limbs of onset predominates that can be generalized until tetra pares progressively increasing over the years and which is usually a long-evolving disease. Diseases of the motor neuron are divided into those that affect the upper and lower, in this case we focus on the first case, which are located inside the cerebral cortex and send axons that form the pyramidal pathway, to later defuse and finally connect to the spinal cord. The manifestations secondary to the lesion of the upper motoneuron are as follows: spastic paralysis, amyotrophy (by disuse), absence of tracing, exalted myotatic reflexes and extensive plantar response.

As it is a disease with a low incidence and few reported cases, it should never be considered as a diagnosis of first instance, having to rule out other differential diagnoses, so over time various diagnostic criteria have been defined to realize the disease, however, as well mentioned, it is a rare disease, so no definitive consensus has been created for the diagnosis and scrutiny of the disease, however, we can lead our diagnostic approach means of any of these, such as the criteria of pringle and cabbage, through a well-established clinic, conducting a thorough clinical history, laboratory studies, ranging from general analyses such as hematic biometry, blood chemistry, functional tests, to cytological examinations of cerebrospinal fluid, quantification of vitamin B12 in serum, trepamic tests, as well as cabinet studies such as electromyography and MRI, where the absence of other pathological entities is verified and in the latter study a marked cortical atrophy of the pre-central turn. Despite the above, it should be noted that the disease may not follow a specific pattern within its natural history, so it may vary the presentation and sequence of them.

\section{Conclusion}

Motor neuron diseases are divided into two groups, and in the case studied, the upper motoneuron is exclusively affected, differing from each other by the form of presentation and clinical manifestations. As it is a rare disease, with a low incidence, multiple differential diagnoses will be considered before concluding in it, considering a diagnosis of exclusion, and thus, a thorough medical history should be made with a proper examination conducting laboratory and cabinet studies relevant to this situation. Although it is a long-term disease with a longer life expectancy than amyotrophic lateral sclerosis, the natural history of the disease will always have a grim outcome, with poor prognosis for life and function, taking into account there is no cure for the disease other than supportive treatment.

\section{References}

1. Tomik B, Anna C, Szczudlik A (2008) Pure primary lateral sclerosis, case reports. Clinical Neurology and Neurosurgery 110(4): 387-391.

2. Jeffrey M, Richard J, Mazen M, Kay M, Mitzumoto H (2015) Primary lateral sclerosis. Neurologic clinics 33(4): 749-760.

3. Agosta F, Canu E, Innugi A, Chio A, Riva N, et al. (2014) Resting state functional connectivity alterations in primary lateral sclerosis. Neurobiol Aging 35(4): 916-925.

4. Meoded A, Morrissette A, Katipally R, Schanz O, Gotts S, et al. (2015) Cerebro-cerebellar connectivity is increased in primary lateral sclerosis. Neuroimage: Clinical 7: 288-296.

5. Santosh R, Rana S (2018) Demographics and clinical characteristics of primary lateral sclerosis: case series and review of literature. Neurodegenerative Disease Management 8(1): 17-23.

6. Martinez M, Leyva R (2001) Primary lateral sclerosis (report of a clinical case and review of clinical manifestations). Mexican Journal of Neuroscience 2(3): 165-167.

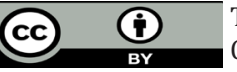

This work is licensed under Creative Commons Attribution 4.0 License

To Submit Your Article Click Here:

Submit Article

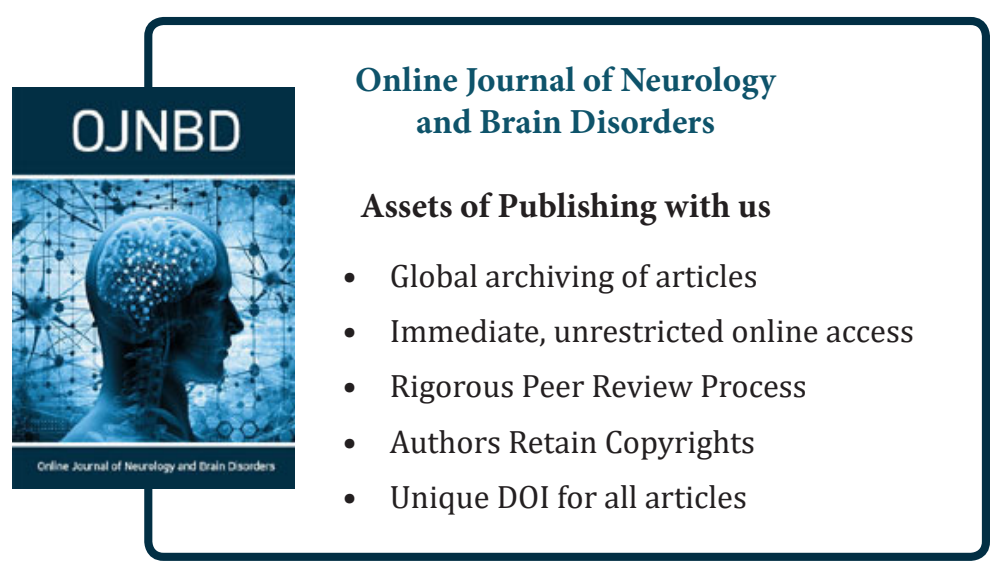

